\title{
CD56 Expression in Normal Immature Granulocytes after Allogeneic Hematopoietic Stem Cell Transplantation
}

\author{
Kazuo Muroi, Shin-ichiro Fujiwara, Raine Tatara, Miyuki Sugimoto, Chihiro Yamamoto, \\ Eisuke Uehara, Akiko Meguro, Kaoru Hatano, Kiyoshi Okazuka, Iekuni Oh, Ken Ohmine, \\ Takahiro Suzuki, Masaki Mori, Tadashi Nagai, and Keiya Ozawa
}

Bone marrow mononuclear cells from 93 patients with hematological malignancies after allogeneic hematopoietic stem cell transplantation (AHSCT) were analyzed using flow cytometry (FCM). The disease was acute myeloblastic leukemia (50 patients), acute lymphoblastic leukemia, and others. Conditioning was myeloablative ( 80 patients) or reduced intensity. The stem cell source was bone marrow (75 patients), peripheral blood stem cells, or cord blood. After AHSCT, granulocyte colonystimulating factor was given to all patients. All patients showed engraftment of the donor cells. FCM was conducted on a median of 22 days after AHSCT. The gate was set around a granulocytic region consisting of immature granulocytes. The positivity rates of $\mathrm{CD} 13, \mathrm{CD} 14, \mathrm{CD} 15, \mathrm{CD} 33, \mathrm{CD} 34, \mathrm{CD} 56$, and HLA-DR in the cells were $59.9 \pm 27.4 \%, 5.8 \pm 8.8 \%, 98.3 \pm$ $1.9 \%, 92.3 \pm 12.4 \%, 2.6 \pm 5.8 \%, 24.3 \pm 16.7 \%$, and $9.1 \pm 6.6 \%$, respectively. The greatest value of CD56 positivity was $73.1 \%$. On the basis of CD56 expression, cases of more than $24 \%$ CD56 positivity were assigned to the CD56-high group (39 patients), while the rest were assigned to the CD56-low/negative group. There were no significant differences between the two groups in terms of disease status, sex, donor, hematopoietic stem cells, days of FCM analysis, or peripheral blood cell counts around the days of performing FCM. These results indicate that CD56 can be expressed in normal immature granulocytes at a variety of expression levels in regenerative bone marrow. Attention should be paid when evaluating aberrant antigen expression of CD56 in granulocytes. 〔 $J$ Clin Exp Hematop 53(3) : 247-250, 2013〕

Keywords: CD56, flow cytometry, granulocyte

\section{INTRODUCTION}

CD56, a neural cell-adhesion molecule, is expressed in normal NK cells, so the antigen is a good marker for identifying malignancies in NK cell lineages. ${ }^{1}$ CD56 is aberrantly expressed in acute myeloblastic leukemia (AML) blasts, especially AML with $\mathrm{t}(8 ; 21)$, for which the prognosis is suggested to be worse than that of CD56-negative AML with $\mathrm{t}(8 ; 21){ }^{2}$ CD56 is regarded as an important marker of aberrant expression in granulocytes of myelodysplastic syndrome and other hematological malignancies. ${ }^{3-5}$ In this report, we show that CD56 can be expressed in normal immature granulocytes in regenerative bone marrow.

\footnotetext{
Received : August 22, 2013

Revised : September 16, 2013

Accepted : October 7, 2013

Division of Cell Therapy, Jichi Medical University Hospital, Shimotsuke, Tochigi, Japan

Corresponding author: Kazuo Muroi, M.D., Division of Cell Therapy, Jichi Medical University Hospital, Shimotsuke, Tochigi, Japan

E-mail: muroi-kz@jichi.ac.jp
}

\section{PATIENTS AND METHODS}

\section{Patients}

Patients admitted to Jichi Medical University Hospital from January 2006 to June 2012 were retrospectively collected (Table 1). All patients had hematological malignancies and received an allogeneic hematopoietic stem cell transplant from a related or unrelated donor after conditioning. Conditioning was either myeloablative or reduced intensity. The former mainly consisted of a standard regimen of cyclophosphamide (CY) plus total body irradiation (TBI) and busulfan (BU) plus CY. In several patients, melphalan (L-PAM) plus TBI was used. The latter were administered as fludarabine (FLD)-based regimens consisting of a combination of FLD, BU plus TBI, FLD, BU plus CY, and FLD, L-PAM plus TBI, similar to a previous report. ${ }^{6}$ Granulocyte colony-stimulating factor (G-CSF) was given to all patients after allogeneic hematopoietic stem cell transplantation (AHSCT). The agent was administered to the patients on day 7 after AHSCT in principle. The date of engraftment was 
Table 1. Patient characteristics

\begin{tabular}{lc}
\hline \multicolumn{1}{c}{ Clinical data } & Patients \\
\hline Age (years, median) & $43(17-67)$ \\
Sex (male/femal, no) & $51 / 42$ \\
Disease (AML/ALL/Others, no) & $50 / 16 / 27$ \\
Status (CR/NCR, no) & $41 / 52$ \\
Conditioning (CY-TBI $\pm a /$ LPAM-TBI/BU-CY/RIST, no) & $54 / 2 / 24 / 13$ \\
Stem cell $(\mathrm{BM} / \mathrm{PB} / \mathrm{CB}$, no) & $75 / 10 / 8$ \\
Donor (related/unrelated, no) & $71 / 22$ \\
G-CSF administration $($ no) & 93 \\
WBC counts $\left(\times 10^{3} / \mu \mathrm{L}\right.$, mean) & $4.8 \pm 4.3$ \\
Hb values $(\mathrm{g} / \mathrm{dL}$, mean) & $8.0 \pm 1.1$ \\
Platelet counts $\times 10^{4} / \mu \mathrm{L}$, mean) & $4.0 \pm 3.0$ \\
FCM performed after AHSCT (days, median) & $22 \pm 6$ \\
\hline
\end{tabular}

AML, acute myeloblastic leukemia; ALL, acute lymphoblastic leukemia; CR, complete remission; NCR, non-complete remission; CY-TBI, cyclophosphamide plus total body irradiation; LPAM-TBI, melphalan plus TBI; BU-CY, busalfan plus $\mathrm{CY}$; RIST, reduced-intensity conditioning; BM, bone marrow; $\mathrm{PB}$, peripheral blood stem cells; $\mathrm{CB}$, cord blood; G-CSF, granulocyte colonystimulating factor; WBC, white blood cells; $\mathrm{Hb}$, hemoglobin; FCM, flow cytometry; AHSCT, hemopoietic stem cell transplantation; no, total number

defined as the first of three consecutive days with an absolute neutrophil count of more than $500 / \mu \mathrm{L}$. Donor chimerism of bone marrow cells was evaluated by fluorescence in situ hybridization using $\mathrm{X}$ - and $\mathrm{Y}$-chromosome probes for sexmismatched pairs or short tandem repeat analysis.

\section{Flow cytometry}

Mononuclear cells were separated from an aliquot of the bone marrow samples and used for flow cytometry (FCM) with a CD45 gate. Along with the course of AHSCT, FCM analysis was performed to identify minimal residual disease as a routine practice. Cells were stained with a fluorescein isothiocyanate-conjugated monoclonal antibody and/or a phycoerythrin-conjugated monoclonal antibody, and peridinin chlorophyll protein-conjugated CD45. Monoclonal antibodies used in this study were as follows: CD11b, CD13, CD14, CD15, CD33, CD34, CD36, CD45, CD56, and HLA-DR. ${ }^{7}$ For the negative controls, cells were stained with isotypematched control antibodies. Initially, FCM was performed to analyze blast phenotypes in the blast region. ${ }^{7}$ In this study, FCM data were reevaluated by setting a gate to identify granulocytes characterized by intermediate CD45 expression and high scatter properties (Fig. 1A). Phenotypes of the cells were analyzed using a flow cytometer (FACSCalibur; BD Biosciences, San Jose, CA). In terms of reanalyzing the FCM data, neither informed consent from each patient nor permission from the Institutional Review Board in our hospital was obtained.

\section{Statistical analysis}

An unpaired t-test and Mann-Whitney U test were used to investigate the differences between two independent populations. Values of $P<0.05$ were considered significant.

\section{RESULTS AND DISCUSSION}

Table 1 shows the characteristics of the patients. Fiftyone were males and 42 were females, and the median age was 43 years (range, 17-67 years). The disease was AML ( $\mathrm{n}=$ $50)$, acute lymphoblastic leukemia $(\mathrm{n}=16)$, myelodysplastic syndrome $(n=13)$, chronic myelogenous leukemia $(n=3)$, diffuse large B-cell lymphoma $(\mathrm{n}=2)$, follicular lymphoma $(\mathrm{n}=2)$, and others $(\mathrm{n}=7)$. Twenty-nine AML patients, 11 acute lymphoblastic leukemia patients, and one mantle cell lymphoma patient were in complete remission, while the others were in non-complete remission, including in chronicphase chronic myelogenous leukemia. Myeloablative conditioning regimens were as follows : $\mathrm{CY}$ plus TBI $(\mathrm{n}=52), \mathrm{CY}$ plus TBI plus cytarabine or etoposide $(\mathrm{n}=2)$, L-PAM plus TBI $(n=2)$, and BU plus CY $(n=24)$. Reduced-intensity conditioning regimens were used for 13 patients. Infused hematopoietic stem cells were from related bone marrow $(\mathrm{n}=$ $11)$, unrelated bone marrow $(n=64)$, related peripheral blood stem cells $(n=11)$, or unrelated cord blood $(n=7)$. The administration of G-CSF was discontinued after engraftment. After engraftment, bone marrow aspiration was performed to confirm engraftment of bone marrow cells and analyze cell phenotypes using FCM. FCM was conducted on a median of 22 days after AHSCT. All patients showed engraftment of the donor cells using the methods described in Materials and Methods. No graft rejection or late-graft failure except for relapse was observed in any of the patients.

As shown in Table 1, peripheral blood leukocytes recovered to within normal ranges in most patients, while red blood cells and platelets showed signs of recovery. Positivity of antigens in granulocytes was as follows (mean \pm SD) : CD11b, $81.1 \pm 11.6 \%$; CD13, $59.9 \pm 27.4 \%$; CD14, $5.8 \pm$ $8.8 \%$; CD15, $98.3 \pm 1.9 \%$; CD34, $2.6 \pm 5.8 \%$; CD36, $8.5 \pm 10.6 \%$; CD56, $24.3 \pm 16.7 \%$; and HLA-DR, $9.1 \pm$ $6.6 \%$ (Fig. 2). The highest and second highest values of CD56-positivity were $73.1 \%$ and $69.6 \%$, respectively. A demonstrable case with CD56-positive granulocytes is shown in Fig. 1. To identify the nature of CD56 expression in the granulocytes, CD56-positive granulocytes were divided into two groups : a CD56-high group (more than $24 \%$ CD56 positivity) and a CD56-low/negative group (equal to or less than $24 \%$ CD56 positivity). The cut-off value of $24 \%$ was introduced on the basis of the mean value of CD56 expression. There were no significant differences between the two groups in disease status (complete remission versus non-complete remission), sex, donor (related versus unrelated), hemato- 

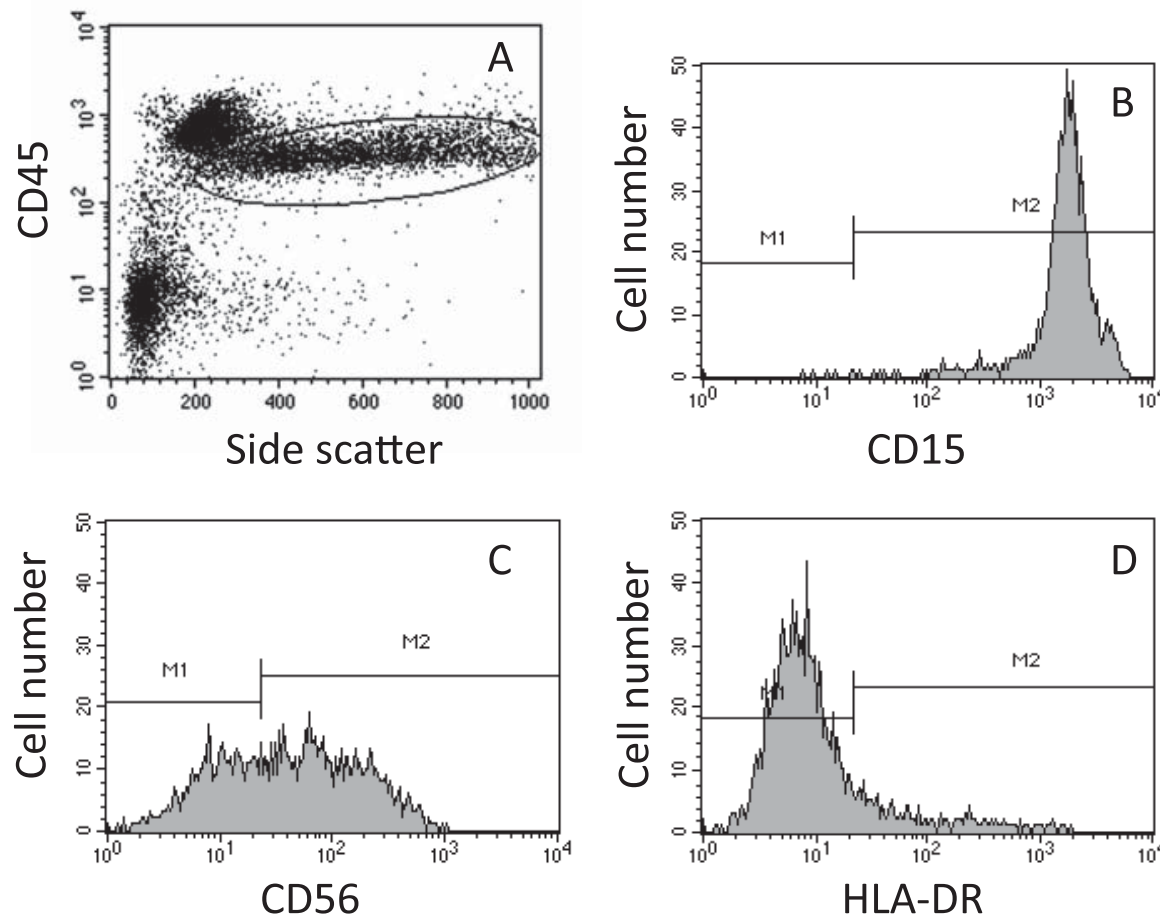

Fig. 1. Flow cytometric analysis of immature granulocytes from a patient after bone marrow transplantation. A gate used for the analysis of immature granulocytes $(1 \mathrm{~A})$ and expression of CD15 (1B), CD56 (1C), and HLA-DR (1D) in the cells.

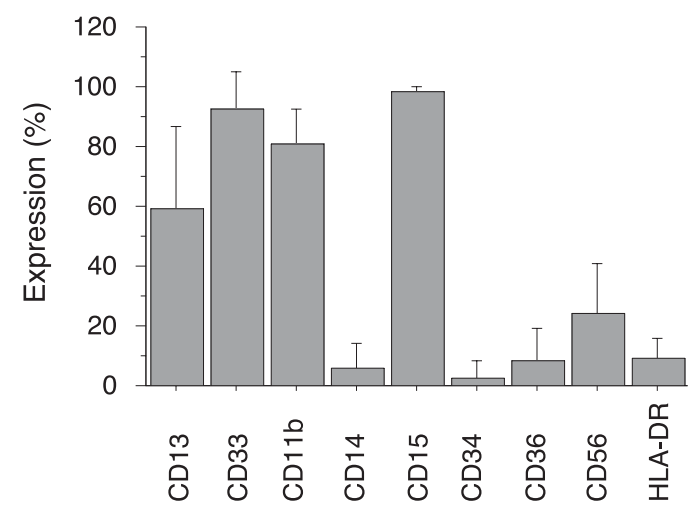

Fig. 2. Antigen expression in immature granulocytes. The data show the mean $\pm \mathrm{SD}$.

poietic stem cell source (bone marrow versus peripheral blood stem cell versus cord blood), conditioning (myeloablative versus reduced intensity), days of FCM analysis, or peripheral blood cell counts around the days of performing FCM. The only significant factor obtained was age : the former group was significantly older (mean, 46.6 years) than the latter (mean, 39.9 years).

Mononuclear cells isolated from bone marrow cells contain lymphocytes, lymphoblasts, erythroblasts, monocytes, myeloblasts, and immature granulocytes. In our study, granulocytes contained in the bone marrow mononuclear cells were promyelocytes, myelocytes, and metamyelocytes because of the high positivity of CD11b, CD15, and CD33, the variable positivity of $\mathrm{CD} 13$, and the negativity of $\mathrm{CD} 14, \mathrm{CD} 34$, and HLA-DR (Fig. 2). ${ }^{8}$ We showed that CD56 can be expressed in normal immature granulocytes at a variety of expression levels in regenerative bone marrow. Patients in our study were given G-CSF, which modulates the antigen expression of neutrophils depending on the time course from administration. ${ }^{9}$ Therefore, CD56 expression in the immature granulocytes may have been due to G-CSF treatment. We did not analyze events associated with AHSCT such as conditioning-related toxicity, graft-versus-host disease, or infections. These events may have influenced CD56 expression in the immature granulocytes in our study. In fact, CD56 may be consistently expressed in some maturation stages in granulocytes. In the future, we should pay attention to evaluating the aberrant antigen expression of CD56 in granulocytes.

\section{REFERENCES}

1 Lanier LL, Le AM, Civin CI, Loken MR, Phillips JH: The relationship of CD16 (Leu-11) and Leu-19 (NKH-1) antigen expression on human peripheral blood NK cells and cytotoxic T lympho- 


\section{Muroi K, et al.}

cytes. J Immunol 136:4480-4486, 1986

2 Baer MR, Stewart CC, Lawrence D, Arthur DC, Byrd JC, et al:: Expression of the neural cell adhesion molecule CD56 is associated with short remission duration and survival in acute myeloid leukemia with $\mathrm{t}(8 ; 21)(\mathrm{q} 22 ; \mathrm{q} 22)$. Blood 90:1643-1648, 1997

3 Wells DA, Benesch M, Loken MR, Vallejo C, Myerson D, et al:: Myeloid and monocytic dyspoiesis as determined by flow cytometric scoring in myelodysplastic syndrome correlates with the IPSS and with outcome after hematopoietic stem cell transplantation. Blood 102:394-403, 2003

4 Chu SC, Wang TF, Li CC, Kao RH, Li DK, et al.: Flow cytometric scoring system as a diagnostic and prognostic tool in myelodysplastic syndromes. Leuk Res 35:868-873, 2011

5 Feng B, Verstovsek S, Jorgensen JL, Lin P: Aberrant myeloid maturation identified by flow cytometry in primary myelofibrosis.
Am J Clin Pathol 133:314-320, 2010

6 Narimatsu H, Kami M, Miyakoshi S, Murashige N, Yuji K, et al.: Graft failure following reduced-intensity cord blood transplantation for adult patients. Br J Haematol 132:36-41, 2006

7 Oka S, Muroi K, Matsuyama T, Sato K, Ueda M, et al.: Correlation between flow cytometric identification of CD33positive cells and morphological evaluation of myeloblasts in bone marrow of patients with acute myeloblastic leukemia. Hematology 14:133-138, 2009

8 Oka S, Nagatsuka Y, Kikuchi J, Yokote T, Hirabayashi Y, et al.: Preferential expression of phosphatidylglucoside along neutrophil differentiation pathway. Leuk Lymphoma 50:1190-1197, 2009

9 Carulli G: Effects of recombinant human granulocyte colonystimulating factor administration on neutrophil phenotype and functions. Haematologica 82:606-616, 1997 